
allemande

51-2| 2019

Les Humanités environnementales : circulations et renouvellement des savoirs en France et en Allemagne

\title{
Environmental Humanities: Wissenstransfer und Wissenserneuerung in Frankreich und Deutschland - Einleitung
}

Aurélie Choné, Tim Freytag, Philippe Hamman et Evi Zemanek

\section{(2) OpenEdition}

\section{Journals}

Édition électronique

URL : https://journals.openedition.org/allemagne/1933

DOI : 10.4000/allemagne.1933

ISSN : 2605-7913

Éditeur

Société d'études allemandes

Édition imprimée

Date de publication : 10 décembre 2019

Pagination : 275-283

ISSN : 0035-0974

Référence électronique

Aurélie Choné, Tim Freytag, Philippe Hamman und Evi Zemanek, „Environmental Humanities: Wissenstransfer und Wissenserneuerung in Frankreich und Deutschland - Einleitung", Revue d'Allemagne et des pays de langue allemande [Online], 51-2 | 2019, Online erschienen am: 02 Dezember 2019, abgerufen am 19 Mai 2021. URL: http://journals.openedition.org/allemagne/1933 ; DOI: https:// doi.org/10.4000/allemagne.1933 


\title{
Dossier: Les Humanités environnementales: circulations et renouvellement des savoirs en France et en Allemagne Environmental Humanities: Wissenstransfer und Wissens- erneuerung in Frankreich und Deutschland - Einleitung
}

\author{
- Aurélie Choné, Tim Freytag, Philippe Hamman \& Evi Zemanek*
}

Seit wenigen Jahren ist im internationalen Rahmen von den Environmental Humanities die Rede, wenn es darum geht, die gemeinsamen Forschungsinteressen von Kultur- und Literaturökologie, Umweltsoziologie, -anthropologie und Humangeographie, Umweltgeschichte und Umweltethik u.a. zu unterstreichen. Für deren gemeinsames, und doch fachspezifisch unterschiedlich gelagertes Interesse an Mensch-UmweltBeziehungen unter den Vorzeichen der ökologischen Krise und für deren interdisziplinäre Zusammenarbeit hat sich nunmehr diese englische Sammelbezeichnung etabliert, die sogleich das französische Äquivalent der Humanités environnementales hervorrief - während sich in Deutschland kein entsprechender eigener Begriff durchgesetzt hat, sondern der englische verwendet wird. Jedoch kann man als Maßstab nicht vollkommen selbstverständlich die Impulse der angloamerikanischen Vorreiter anlegen, obwohl sie in mancher Hinsicht wegweisend waren. Vor diesem Hintergrund wird in diesem Band ein anderer Ansatz verfolgt. Denn es geht darum, verschiedene Ausprägungen der ,Environmental Humanities' oder ,Humanités environnementales' aus den Perspektiven der französisch- und deutschsprachigen Forschung zu beleuchten sowie diese Wissenschafts- und Wissenskulturen $\mathrm{zu}$ vergleichen und $\mathrm{zu}$ vermitteln.

\footnotetext{
* Aurélie Choné, Dozentin für Neuere Deutsche Literatur und Ideengeschichte am Institut für Germanistik der Universität Strasbourg; Tim Freytag, Professor für Humangeographie an der Albert-Ludwigs-Universität Freiburg; Philippe Hamman, Professor für Stadt- und Umweltsoziologie am Institut für Stadtsoziologie und regionale Raumplanung der Universität Strasbourg; Evi Zemanek, Professorin für Medienkulturwissenschaft an der Albert-Ludwigs-Universität Freiburg.
} 
Mit dem Etikett ,Humanités environnementales` möchte man den Geisteswissenschaften eine gesellschaftliche Relevanz zusprechen, die sie zweifellos in Anspruch nehmen können und heutzutage auch für ihre eigene Legitimation benötigen, und man möchte damit auch die vielseitigen interdisziplinären Kooperationsmöglichkeiten mit den Gesellschafts- und neuerdings auch mit den Umweltwissenschaften aufzeigen. Signalisiert das ,environmental' den Fokus auf Umweltprobleme, und zwar speziell auf deren kulturelle Dimension, so halten die ,Humanities' zunächst weitgehend an einem geisteswissenschaftlich geprägten interpretatorischen Zugriff fest, zeigen sich dabei aber offen für empirische Ansätze. Der brisanten aktuellen Debatte über verschiedenste Aspekte des Mensch-Natur-Verhältnisses können die Environmental Humanities, so ihr Angebot an die Umwelt- und Naturwissenschaften, „konzeptuelle Schärfe, moralisches Gewicht und eine historische Tiefendimension "verleihen ${ }^{(1)}$.

Auf mehrere Essays, welche die Environmental Humanities konzeptuell und in methodischer Hinsicht ${ }^{(2)}$ betrachten, und diverse neu gegründete Netzwerke, digitale Plattformen und institutionell verankerte Zentren folgten die ersten Sammelbände mit dem Ziel, das breite Spektrum der beteiligten Disziplinen und deren Ansätze vorzustellen. Dazu gehört auch der von Aurélie Choné, Isabelle Hajek und Philippe Hamman herausgegebene Guide des Humanités environnementales ${ }^{(3)}$, der eine Vielzahl von geistes- und gesellschaftswissenschaftlichen Fächern versammelt.

Seit einigen Jahren stehen Vertreter und Vertreterinnen der Humanités environnementales der Universität Strasbourg und der Universität Freiburg miteinander in einem engen Dialog, der unter anderem im Rahmen des Oberrheinischen Cluster für Nachhaltigkeitsforschung ${ }^{(4)}$ stattfindet und im CIERA-Programm (Centre interdisciplinaire d'études et de recherches sur l'Allemagne) gefördert wird unter dem Titel „Circulations et renouvellement des savoirs sur la nature et l'environnement en France et en Allemagne / Natur und Umwelt: Wissenstransfer und Wissenserneuerung in Frankreich und Deutschland“. Aus den dank dieser Förderung veranstalteten Workshops in Strasbourg und Freiburg (2016-2018) ist der vorliegende Band hervorgegangen.

Der Band umfasst sechs literaturwissenschaftliche und fünf gesellschaftswissenschaftliche Beiträge: darunter sind solche, die selbst schon den Vergleich zwischen Diskursen, Ansätzen und Disziplinen in den deutsch- und französischsprachigen Kulturräumen ziehen; andere Beiträge, die nur die Situation auf der jeweils einen Seite des Rheins beleuchten, laden die Leser und Leserinnen dazu ein, die Vergleiche und Verbindungen zu ziehen. Paar- und gruppenweise greifen die Beiträge zentrale

1 Sabine Wilke, „Environmental Humanities“, in: Gabriele Dürbeck und Urte STOBbe (Hg.), Ecocriticism: Eine Einführung, Köln, Böhlau, 2015, S. 94-106, hier S. 102.

2 Siehe Florence Rudolf (Hg.), „Humanités environnementales - Quoi de neuf du côté des méthodes?“, Sonderheft der Revue d'Allemagne et des Pays de langue allemande, 50/2 (2018), S. 227-237.

3 Aurélie Choné, Isabelle Hajex und Philippe Hamman (Hg.), Guide des Humanités environnementales, Villeneuve d'Ascq, Presses universitaires du Septentrion, 2016. Siehe auch Aurélie Choné, Isabelle Hajex und Philippe Hamman (Hg.), Rethinking Nature. Challenging Disciplinary Boundaries, London/New York, Routledge, 2017.

4 Am Oberrheinischen Cluster für Nachhaltigkeitsforschung sind seit 2016 die Universitäten von Eucor - The European Campus in Basel, Freiburg, Karlsruhe, Mulhouse und Strasbourg, die Universität Landau sowie verschiedene Fachhochschulen als assoziierte Partner beteiligt. Siehe www.nachhaltigkeit-oberrhein.info/de/home. 
Diskurse und Themen der Environmental Humanities auf. Zwei Beiträge widmen sich der Anthropozän-Debatte (Dürbeck, Thober); zwei andere Texte diskutieren deutsche, französische und auch anglophone Ansätze einer ökologisch orientierten Literaturwissenschaft (Choné, Zemanek); weitere zwei Beiträge befassen sich mit dem jungen, florierenden Forschungsfeld der Animal Studies (Hamm, Hecht). Daran knüpft thematisch ein sozialwissenschaftlicher Beitrag zur Wiedereinführung des Luchses (Christen) an, gefolgt von Überlegungen zum Konzept der Solidarischen Landwirtschaft (Zwer). Zwei weitere Texte untersuchen die Wissenszirkulation zwischen Deutschland und Frankreich: in einem Fall diejenige des Nachhaltigkeitswissens im Allgemeinen (Hamman), im anderen Fall diejenige des konkreten Wissens über Passivhäuser (Mangold), bevor abschließend ein humangeographischer Beitrag die Folgen des Rückbaus von Kernkraftwerken diskutiert (Kramer). Im Folgenden werden die einzelnen Beiträge kurz vorgestellt.

Gabriele Dürbeck befasst sich in ihrem Beitrag mit Narrativen des Anthropozän in deutschsprachigen Qualitätszeitungen, die zwischen 2010 und 2016 erschienen sind. In ihrem Text skizziert die Literatur- und Kulturwissenschaftlerin zunächst, wie sich der interdisziplinäre Anthropozändiskurs seit dem Jahr 2000 zusehends etabliert hat. Die Ausführungen richten sich auf die zeitliche Einordnung des Beginns des Anthropozän sowie auf fünf verschiedene Narrative, die als prägend für das Anthropozän angesehen werden können. Zu diesen Narrativen zählen u.a. das Katastrophennarrativ und das Narrativ der Dependenz von Natur und Kultur. Im empirischen Teil ihres Beitrags arbeitet Dürbeck heraus, inwiefern sich die Frankfurter Allgemeine Zeitung (FAZ) bzw. Frankfurter Allgemeine Sonntagszeitung (FAS), die Süddeutsche Zeitung (SZ), die Neue Zürcher Zeitung (NZZ) und die tageszeitung (taz) in ihrer Berichterstattung über das Anthropozän voneinander unterscheiden und welche der identifizierten Narrative dabei im Vordergrund stehen.

Im Mittelpunkt des Beitrags von Benjamin Thober steht das Anthropozoikum-Konzept, das von Hubert Markl während der 1980er Jahre im Zusammenhang mit einer Problematisierung des Artensterbens vorangebracht wurde. Thober arbeitet heraus, inwieweit das Anthropozoikum-Konzept als ein Vorläufer der Idee des Anthropozän interpretiert werden kann. Durch diese historisch geleitete Betrachtung wird deutlich, dass die Konzepte des Anthropozoikums und des Anthropozän jeweils zeit- und kontextgebunden erscheinen. In seinen Ausführungen stützt sich Thober auf eine Reihe ausgewählter Texte von Markl. Im Einzelnen wird zunächst nach der wissenschaftlichen Grundlegung des Anthropozoikums (im Unterschied zum Anthropozän) gefragt, bevor sich Thober politisch-ökonomischen Aspekten im Spannungsfeld zwischen den Grenzen des Wachstums und der Ökologischen Modernisierung zuwendet und sich darüber hinaus auch mit dem naturalistisch geprägten Menschenbild des Anthropozoikums kritisch auseinandersetzt und dieses in Anlehnung an die Philosophische Anthropologie von Helmuth Plessner hinterfragt. Abschließend werden vorhandene Bezüge zum aktuellen Anthropozän-Diskurs hergestellt.

Aurélie Choné befasst sich in ihrem Beitrag mit der Herausbildung von umweltbezogenen Literaturwissenschaften in Deutschland und Frankreich. Indem sie für Deutschland und Frankreich die jeweils charakteristischen Forschungsansätze beleuchtet und deren Entwicklungslinien herausarbeitet, verdeutlicht Choné 
vorhandene Gemeinsamkeiten und Anknüpfungspunkte. Damit entwirft sie eine Grundlage für eine künftige Entfaltung einer deutsch-französischen Komparatistik im Forschungsbereich von „Literatur und Ökologie“. In ihren Ausführungen richtet die Germanistin und Ideengeschichtlerin ihren Blick zunächst darauf, wie der Ecocriticism im US-amerikanischen Kontext als Forschungsfeld entstanden ist, um dann später auf die Entwicklungen in Deutschland und Frankreich einzugehen. Dabei identifiziert sie für beide Sprachräume zahlreiche Forschungsansätze, Referenzwerke und Persönlichkeiten, die sie einordnet und zueinander in Beziehung setzt. Abschließend geht sie auf Verflechtungen und Anknüpfungspunkte ein, die eine geeignete Grundlage für eine komparatistische Herangehensweise in den umweltbezogenen Literaturwissenschaften bilden können.

Der Beitrag von Evi Zemanek zeigt auf, wie durch ökologische Transformationen sowohl poetische Verfahren als auch literarische Gattungen maßgeblich geprägt werden und somit Veränderungen des literarischen Gattungssystems eintreten können. Ergänzend zum Ansatz des Ecocriticism stellt Zemanek zunächst den Ansatz einer an der Kulturökologie orientierten Literaturökologie vor und erläutert, wie diese für Untersuchungen mit einem Fokus auf Gattungspoetik nutzbar gemacht werden kann. Für den neuen Dorfroman wird dies exemplarisch anhand der Romane Unterleuten von Juli Zeh (2016) und Vor dem Fest von Saša Stanišić (2014) unter Rückgriff auf Forschungsansätze der Literaturökologie, der Akteur-Netzwerk-Theorie und des New Materialism herausgearbeitet.

Elisabeth Hamm verdeutlicht mit ihrem Beitrag, wie sich ein literarischer Text aus dem Blickwinkel der Animal Studies betrachten lässt. Diesen besonderen Zugang arbeitet sie am Beispiel der Rolle des Pferdes in den Jahrmarktszenen in Georg Büchners Woyzeck detailliert heraus. Im Unterschied zu klassischen Interpretationsansätzen, die das Pferd vor allem als Symbol oder Motiv erkennen, erlauben die Cultural and Literary Animal Studies ein neues Verständnis der Inszenierung von Tieren in der Literatur. So erkennt Hamm das Pferd als einen Träger von kulturellem und literarischem Wissen über Pferde bzw. Bühnenpferde im zeitgenössischen Kontext zu Anfang des 19. Jahrhunderts. Sie verdeutlicht in ihren Ausführungen, wie sich im Pferd auch die damaligen wissenschaftlichen, anatomischen und philosophischen Diskurse widerspiegeln. Durch den Aufsatz wird deutlich, wie die Animal Studies dazu beitragen können, literarische bzw. theatralische Gegenstände auf eine neue Art und Weise zu fassen sowie die Geistes- und Literaturwissenschaften neu zu ordnen.

Im Mittelpunkt des Beitrags von Stefan Hecht steht die sprachliche Konstruktion humanimaler Emotionen, die er als zwischen Menschen und Tieren angesiedelt konzeptualisiert und am Beispiel des Geschichtenbands Die Bärin von Otto Alscher herausarbeitet. Zunächst erläutert Hecht in Grundzügen, wie in der wissenschaftlichen Disziplin der Psychologie Emotionen erfasst und in Bezug auf verschiedene Dimensionen und Komponenten klassifiziert werden können. Vor diesem Hintergrund wendet er sich dann einzelnen Geschichten über die Bärin zu und zeigt auf, wie eine sprachliche Konstruktion von Emotionen erfolgt, die dem Tier zugeschrieben werden. Dabei geht Hecht insbesondere auf die raumsemantische, kinetische und psychoakustische Gestaltung ein. Weiterhin erörtert er die Bedeutung von moralischen und existentiellen Emotionen. Im Ganzen verdeutlichen die Ausführungen, wie die 
Perspektiven von Menschen und Tieren als gleichwertig angesehen werden können und wie sich die Emotionen von Menschen und Wildtieren einander annähern und möglicherweise sogar miteinander verschmelzen.

Guillaume Christen befasst sich in seinem Beitrag mit dem LIFE-Projekt zur Wiedereinführung des Luchses im deutsch-französischen Biosphärenreservat Pfälzerwald/Nordvogesen. Im Mittelpunkt steht dabei die Frage nach den möglichen Auswirkungen der Wiedereinführung von Raubtieren auf den Umgang mit der Natur. In seiner soziologischen Untersuchung stützt sich Christen auf eine Befragung von Jägern und Förstern. Er arbeitet das Selbstverständnis von Jägern heraus, das die Jagd als eine Aktivität zur Pflege und dauerhaften Bewahrung des Wildbestands erachtet. Weiterhin erörtert Christen, inwiefern die Wiedereinführung des Luchses zwar keine unmittelbare Veränderung der Wissensbestände im Hinblick auf den Umgang von Jägern mit der Natur bewirke, aber dennoch zu einer Marginalisierung und Infragestellung ihres historisch gewachsenen Selbstverständnisses führen könne.

Im Beitrag von Nepthys Zwer geht es um Aktivitäten für eine Solidarische Landwirtschaft. Zunächst erläutert Zwer die Idee einer Agrarwende in Deutschland als Gegenentwurf zur industriellen Landwirtschaft und das Konzept der Solidarischen Landwirtschaft, das Ähnlichkeiten, aber auch Unterschiede gegenüber dem in Frankreich bekannten Konzept der AMAP (Association pour le maintien d'une agriculture paysanne) aufweist. Am Beispiel der SoLaVie Ortenau und der nicht-gewinnorientierten Organisation Teikei Coffee arbeitet die Germanistin und Kulturhistorikerin heraus, wie sich das Konzept der Solidarischen Landwirtschaft umsetzen lässt. Sie erörtert, welchen Beitrag diese Initiativen mit den darin aktiven Menschen und den Verbraucherinnen und Verbrauchern leisten können, um die Agrarwende sowohl im lokalen Umfeld wie auch im globalen Rahmen zu verwirklichen.

Im Mittelpunkt des Beitrags von Philippe Hamman steht das Wissen über Nachhaltigkeit in Deutschland und Frankreich. Ausgehend von der Überlegung, dass geistesund sozialwissenschaftliche Forschungsarbeiten und Debatten über Nachhaltigkeit nicht nur den aktuellen Stand der Forschung zum Ausdruck bringen, sondern auch den Stand entsprechender Diskussionen in Gesellschaft und Politik wiedergeben, nimmt der Soziologe einen Vergleich anhand einer Auswahl von Handbüchern und besonders herausragenden wissenschaftlichen Beiträgen vor, die in Deutschland und Frankreich seit den 2000er Jahren veröffentlicht wurden. Es handelt sich um eine differenzierte Analyse darüber, welche nachhaltigkeitsbezogenen Begrifflichkeiten in diesem Bereich in Deutschland und Frankreich wissenschaftlich verwendet werden, was diese auszeichnet und welche Veränderungen diesbezüglich während der vergangenen Jahre aufgetreten sind. Hinsichtlich der Zirkulation von Nachhaltigkeitswissen wird deutlich, dass einer neuen Konzeptualisierung der Interaktionen zwischen Umwelt und Gesellschaft sowie den Möglichkeiten für einen praktischen Anwendungsbezug der Forschungsarbeiten und deren gesellschaftliche Umsetzung eine zunehmende Bedeutung zugeschrieben wird.

Marie Mangold wendet sich in ihrem Beitrag dem Energiestandard des Passivhauses zu und untersucht die Verbreitung von Passivhaus-Modellen und dem damit verbundenen Wissen in Deutschland und Frankreich. Die Soziologin zeichnet nach, wie diese international als Modell anerkannte und zertifizierte Form des nachhaltigen Bauens 
seit den 1990er Jahren in Deutschland entwickelt und während des vergangenen Jahrzehnts zunehmend auch in Frankreich aufgegriffen wurde. Gestützt auf eigene Feldforschung einschließlich zahlreicher Experteninterviews und anderer Quellen analysiert Mangold die Entwicklung des elsässischen Marktes für Passivhäuser während der vergangenen Jahre. Abschließend greift sie die Frage auf, welche Probleme und Kontroversen im Zusammenhang mit der lokalen Umsetzung von international anerkannten Standards verbunden sein können.

Der Beitrag von Caroline Kramer handelt von den sozialen und wirtschaftlichen Folgen des Rückbaus von Kernkraftwerken. Anhand aktueller Beispiele aus BadenWürttemberg und auf der Grundlage von Befragungen untersucht die Humangeographin, wie die betroffene Bevölkerung und Experten aus den Gemeinden mit den Veränderungen infolge des Rückbaus eines Kernkraftwerks umgehen. Kramer arbeitet heraus, dass trotz einer kritischen Haltung zur Kernkraft weitgehend darauf vertraut wird, dass der Rückbau sicher vollzogen wird. Anlass zur Sorge bietet vor allem die Befürchtung, dass der eigene Standort vom derzeitigen Zwischenlager zum Endlager werden könnte. Aus der Sicht der Befragten hängen die Zukunftsperspektiven der Gemeinde als Wohn- und Wirtschaftsstandort entscheidend davon ab, ob es gelingt, den Rückbau rasch und sicher zu vollziehen, den wirtschaftlichen Strukturwandel langfristig voranzutreiben und Arbeitsplätze zu schaffen sowie vorhandene Stärken der Gemeinde auch weiterhin zu nutzen.

Zum Schluss können die eingangs formulierten Fragestellungen noch einmal aufgegriffen werden: Inwiefern lassen sich all diese Ansätze und Beiträge aus unterschiedlichen disziplinären Feldern der Literatur-, Geistes- und Sozialwissenschaften (u.a. Kulturwissenschaft, Soziologie, Humangeographie und Germanistik) unter dem gemeinsamen Dachbegriff der ,Environmental Humanities' versammeln? Besitzt dieses Konzept in Deutschland und Frankreich überhaupt die gleiche Bedeutung? Bringt es einen Paradigmenwechsel bei der Behandlung umweltbezogener Themen im Bereich der Geistes- und Gesellschaftswissenschaften zum Ausdruck? Wie wirken die Humanities auf die Frage nach dem Mensch-Umwelt-Verhältnis und umgekehrt wie wirkt diese Frage auf die Humanities?

Aus dem vorliegenden Band geht hervor, dass sowohl in Frankreich als auch in Deutschland der Ausdruck ,Environmental Humanities' dazu dient, jene sich herausbildenden Forschungsansätze zusammenzufassen, die ein neues Verständnis des Natur-Kultur-Verhältnisses anstreben sowie eine stark ethische und handlungsbezogene Dimension aufweisen. Die Forschungsfelder, die sich mehr oder weniger explizit auf die ,Environmental Humanities' berufen, messen auch interkultureller sowie interund transdisziplinärer Zusammenarbeit eine große Bedeutung zu. In der Tat zeugen diese Forschungsfelder von einer starken Interdisziplinarität und können sich gegenseitig nicht ignorieren. Literaturwissenschaftler und Literaturwissenschaftlerinnen können nicht mehr nur auf Literatur oder Sozialwissenschaftlerinnen und Sozialwissenschaftler auf Gesellschaft fokussieren, sondern sie müssen auch Wissensbestände aus anderen Disziplinen wie Biologie, Ökologie, Ethologie, Emotionsforschung usw. berücksichtigen und einbeziehen. Die Umweltproblematik wirkt so strukturierend, dass sie neue Forschungsfelder ins Leben gerufen hat. 
Die interkulturelle Dimension, und hier genauer der deutsch-französische Vergleich, darf auch nicht vernachlässigt werden, weil unterschiedliche kulturelle Traditionen in der Entwicklung dieser Forschungsfelder eine große Rolle spielen, wie es Choné und Hamman jeweils im Bereich der Literatur- und Kulturwissenschaften und der Sozialwissenschaften herausarbeiten. Solche vergleichenden Analysen erfordern eine fundierte Kenntnis beider Kulturgeschichten und eine weitreichende historische Perspektive. Das gilt auch für Forscher und Forscherinnen, die im Bereich grenzüberschreitender Arbeit tätig sind (Christen, Zwer).

Die Frage nach den Forschungsgebieten der Environmental Humanities im französischen und deutschen Kontext ist umso interessanter, als die beiden „nationalen Traditionen“ der Beziehung zur Natur als sehr unterschiedlich gelten: als Erbe der Aufklärung in Frankreich, als Erbe der Romantik in Deutschland. Jedoch waren die historischen Prozesse kontrastreicher: das Natur-Deutschland dem kartesischen Frankreich gegenüberzustellen, ist eine vereinfachende Annahme. Die Aufklärung hat nämlich auch andere Wege gesucht (siehe z.B. René Descartes' Traité des passions de l'âme von 1649) und die französische Romantik war selbst eine wichtige Bewegung (siehe u.a. ihren Vertreter François-René de Chateaubriand), die sogar das amerikanische Nature writing beeinflusste. Andererseits spielte die deutsche Wissenschaft seit dem Kaiserreich eine Vorreiterrolle (der erste Nobelpreis für Physik ging 1901 an Wilhelm Conrad Röntgen; 1911 wurde in Berlin die Kaiser-Wilhelm-Gesellschaft zur Förderung der Wissenschaften, die heutige Max-Planck-Gesellschaft, gegründet usw.).

Angesichts der aktuellen Fragen zur Wahrnehmung von Natur und Umwelt und nach den wechselseitigen Abhängigkeiten von Kultur und Natur ist der Vergleich besonders interessant: enthüllen die Environmental Humanities diese konzeptuelle Spaltung oder setzen sie diese fort? Diese Ausgabe der Revue d'Allemagne versammelt diesbezüglich mehrere sich ergänzende Ansätze.

Um die Existenz zweier unterschiedlicher Traditionen zu erklären, muss einerseits die Genealogie des Wissens über die Natur in beiden Ländern rekonstruiert werden. Alle Beiträge gehen dieser Frage nach und analysieren sie unter drei zusammenhängenden Gesichtspunkten. Will man die Entstehung der Environmental Humanities in Frankreich und Deutschland untersuchen, muss man sich zunächst mit Herkunft und Wandel der Begriffe auseinandersetzen: Anthropozän, Ökokritik, Ökologie, Wende, Nachhaltigkeit, Postwachstum usw. Es geht darum, aus einer Vielzahl von Indizien und Spuren diese Konzepte herauszuarbeiten: sei es auf dem Gebiet der Literatur (Thober, Choné, Zemanek, Hamm, Hecht), der Presse (Dürbeck) oder der Handbücher (Hamman), der sozialen Praktiken der Jagd (Christen), der Ernährung (Zwer), des Wohnens (Mangold) oder der Energie (Kramer). Dies ermöglicht in einem zweiten Schritt, die Erzeugung von Wissen zu hinterfragen. Soll man die Ökokritik als eine neue literarische Auseinandersetzung mit der Natur ansehen, vom ecocriticism bis hin zu den nature-culture studies (Choné, Zemanek)? Wie helfen die animal studies, das ,Wissen' über die Natur zu überdenken (Hamm, Hecht, Christen)? Während nachhaltige Entwicklung und Nachhaltigkeit seit Mitte der 1980er Jahre durchaus erfolgreich sind und auf akademischer Ebene, entweder als Objekt (einschließlich um sie einer kritischen Prüfung zu unterziehen) oder als Paradigma, aufgenommen wurden, stellt sich die Frage der damit verbundenen sozialen Praktiken und Neuinterpretationen 
von Umwelt- und Naturfragen in Frankreich und Deutschland (Christen, Zwer, Hamman, Mangold, Kramer). In einem dritten Schritt wird es möglich, sich der Frage der Vermittlung und den Vermittlerinnen und Vermittlern zu widmen. Dies trifft auf die Literatur zu: So wird Hubert Markls Anthropozoikum-Konzept von Benjamin Thober in Bezug auf aktuelle Debatten über das Anthropozän analysiert; man kann auch die Begriffe von décroissance und Postwachstum erwähnen, die sich, je nach Sprache, in Frankreich und Deutschland nicht vollständig überlappen (Hamman). Dies ist auch aus Sicht der sozialen Praktiken auffällig; es ist hier notwendig, eine Vielzahl sozialräumlicher Skalen zu berücksichtigen. Marie Mangold zeigt, wie die deutschen Standards des Passivhaus Instituts in Frankreich beim Bau von Häusern mit hoher Energieeffizienz eingesetzt werden. Gleichzeitig wird die „Regionalisierung“ dieser Fragen hervorgehoben, wenn im Elsass ein Gewerbeverband für Passivbau entwickelt wird, der sich von einer anderen nationalen Struktur abhebt und ein hybrides Referenzsystem schafft. Der Artikel von Guillaume Christen bestätigt dies am Beispiel der Wiedereinführung des Luchses und zeigt unterschiedliche Prozesse und Reaktionen zwischen lokalen Akteuren (einschließlich Jägern) in den beiden nationalen Teilen desselben Regionalparks, nämlich dem grenzüberschreitenden Biosphärenreservat Nordvogesen/Pfälzerwald.

Andererseits ermöglichen diese Genealogie des Wissens und die Berücksichtigung dieser diachronen Dimension, auch der aktuellen Entwicklung von Natur- und Umweltkonzepten besser zu folgen, sowohl im Hinblick auf die Ideengeschichte und der cultural studies als auch hinsichtlich der empirischen und kritischen Grundlagen der Sozialwissenschaften. Dies stellt binäre Interpretationsansätze in Frage. Philippe Hamman zeigt am Konzept der Nachhaltigkeit, wie es in Frankreich und Deutschland gehandhabt wird. Einerseits wirft dies Fragen zu Paradigmen und Neugestaltungen von Fachbereichen auf: Man kann insbesondere an die Unterschiede und Zusammenhänge zwischen Umweltsoziologie und Risikosoziologie denken, aber auch an den Platz der kritischen Soziologie bzw. an die Bewegung der ökologischen Modernisierung auf beiden Seiten des Rheins. Es ergeben sich auch in Bezug auf die untersuchten Objekte unterschiedliche Methoden (insbesondere in der sozialwissenschaftlichen Literatur) zwischen den Lehrbüchern und spezialisierteren oder engagierteren Werken, die jeweils unterschiedliche Diffusionswege und Porositäten aufweisen.

Es ist also wichtig, die gleichzeitigen Forschungen zu verwandten Fragestellungen und ihre gegenseitige Rezeption in den Blick zu nehmen - und zwar nicht nur, weil Internationalisierung gefragt ist, sondern weil es letztlich darum geht, ökologische Probleme, die transnational und global sind, zu begreifen und gemeinsam Lösungsansätze zu entwickeln. Die Zirkulation des Wissens sollte daher einen zentralen Platz einnehmen.

Dabei wird auch die Bedeutung der anglophonen Sphäre für diesen Wissenstransfer deutlich, weil diese eine besondere Rolle im globalisierten Austauschmechanismus einnimmt; dies spiegeln die bibliographischen Angaben in mehreren Beiträgen wider (insbesondere Dürbeck und Choné, aber auch Thober und Zemanek). Fragen aus der angloamerikanischen Forschung sind in Frankreich und Deutschland im Umlauf, ohne dass dies auf irgendeinen „Vormarsch“ oder eine „Verzögerung“ hindeutet. Zum Beispiel wurde vor allem durch die angloamerikanische Forschung zum Nature 
writing der Ecocriticism bzw. die Ecocritique nach Frankreich importiert (Choné). Auch die zwischen deutschen Forschenden und angloamerikanischen Netzwerken geknüpften Verbindungen tragen zu diesem Wissenstransfer bei, wie z.B. das von der Alexander von Humboldt-Stiftung unterstützte Environmental Humanities Transatlantic Research Network ${ }^{(5)}$, an dem Gabriele Dürbeck und Evi Zemanek beteiligt sind. Eine einseitige Fokussierung einer einzelnen Forschungskultur wäre nicht sehr zielführend, um einen Bereich zu erkunden, der die klassischen disziplinären Grenzen sprengt. So lautet die Lektion dieses Bandes, und dies in jedem der drei Bereiche: Ökokritik bzw. Literaturökologie, animal studies und Nachhaltigkeitsforschung ${ }^{(6)}$.

Deshalb verlangt diese Ausgabe der Revue d'Allemagne von den Forschenden eine kritische Reflexion über das eigene Denken und über die Aufforderung zur Produktion eines „systemischen“, „komplexen“ und „integrierten“ Wissens, wie sie bei der Erforschung der Wechselwirkung zwischen Naturwissenschaften und Sozialwissenschaften seit der letzten Hälfte des 20. Jahrhunderts gilt ${ }^{(7)}$. Ohne zu versuchen, eine erschöpfende und vereinheitlichte Synthese der bestehenden Arbeiten zu präsentieren, möchten wir über die Neukonfiguration dieser Disziplinen und ihrer Objekte in der aktuellen Produktion interdisziplinärer Fragestellungen berichten; dies ist ein weiteres Merkmal der hier versammelten Beiträge, und dies gilt für beide Sprachen.

Bleibt die Herausforderung für die Environmental Humanities, sich nicht mit der (wenn auch unerlässlichen ${ }^{(8)}$ ) wissenschaftlichen Reflexion zu begnügen, sondern Forschung und Handeln miteinander zu verknüpfen: Wie lebt man heute mit der Natur ${ }^{(9)}$ ?

Der vorliegende Band legt den Akzent auf vier Themenfelder: das Anthropozänkonzept (Dürbeck, Thober), die Kultur- und Literaturökologie (Choné, Zemanek), die Frage nach Mensch-Tier-Relationen (Hamm, Hecht, Christen) und die Nachhaltigkeitsproblematik (Zwer, Hamman, Mangold, Kramer), wobei anhand von Beispielen aufgezeigt wird, wie sich diese Themenbereiche sukzessive innerhalb der Environmental Humanities herausgebildet und miteinander verbunden haben, um neue Forschungsfelder aufzuspannen - und zwar Anthropocene Studies, Ecocriticism/ Ökokritik, Animal Studies und Sustainability Studies - die miteinander kommunizieren und zueinander vielfältige Brücken schlagen. Eben diese Brücken bringen zum Ausdruck, wie die ,Environmental Humanities' funktionieren und weshalb sich ein all diese Ansätze zusammenführender ,Umbrella-Term 'als notwendig erwiesen hat.

5 environmentalhumanitiesnetworkdotorg.wordpress.com.

6 Siehe auch Ursula KLuwick und Evi Zemanek (Hg.), Nachhaltigkeit interdisziplinär. Konzepte, Diskurse und Praktiken. Ein Kompendium, Köln, Böhlau, 2019.

7 Siehe Nicole Mathieu und Marcel Jollivet (Hg.), Du rural à l'environnement. La question de la nature aujourd'hui, Paris, L'Harmattan, 1989.

8 Siehe Guillaume Blanc, Élise Demeulenaere und Wolf Feuerhahn (Hg.), Humanités environnementales. Enquêtes et contre-enquêtes, Paris, Publications de la Sorbonne, 2017.

9 Choné/Hajek/Hamman (Hg.), Rethinking Nature (note 3). 